OPEN ACCESS

Edited by:

Roberta Tasso,

University of Genoa, Italy

Reviewed by:

Jeroen Van De Peppel,

Erasmus Medical Center, Netherlands

Hanna Taipaleenmäki,

University Medical Center Hamburg-

Eppendorf, Germany

Garnier Delphine,

INSERM U1138 Centre de Recherche

des Cordeliers (CRC), France

${ }^{*}$ Correspondence:

Alfredo Cappariello

a.cappariello@gmail.com

Specialty section:

This article was submitted to

Molecular and Cellular Oncology,

a section of the journal

Frontiers in Oncology

Received: 09 June 2021

Accepted: 26 August 2021

Published: 20 September 2021

Citation:

Cappariello A and Rucci N (2021) Extracellular Vesicles in Bone Tumors:

How to Seed in the Surroundings

Molecular Information for Malignant

Transformation and Progression.

Front. Oncol. 11:722922.

doi: 10.3389/fonc.2021.722922

\section{Extracellular Vesicles in Bone Tumors: How to Seed in the Surroundings Molecular Information for Malignant Transformation and Progression}

\author{
Alfredo Cappariello * and Nadia Rucci \\ Department of Biotechnological and Applied Clinical Sciences, University of L'Aquila, L'Aquila, Italy
}

Bone is a very dynamic tissue hosting different cell types whose functions are regulated by a plethora of membrane-bound and soluble molecules. Intercellular communication was recently demonstrated to be also sustained by the exchange of extracellular vesicles (EVs). These are cell-derived nanosized structures shuttling biologically active molecules, such as nucleic acids and proteins. The bone microenvironment is a preferential site of primary and metastatic tumors, in which cancer cells find a fertile soil to "seed and blossom". Nowadays, many oncogenic processes are recognized to be sustained by EVs. For example, EVs can directly fuel the vicious cycle in the bone/bone marrow microenvironment. EVs create a favourable environment for tumor growth by affecting osteoblasts, osteoclasts, osteocytes, adipocytes, leukocytes, and endothelial cells. At the same time other crucial tumor-mediated events, such as the premetastatic niche formation, tumor cell dormancy, as well as drug resistance, have been described to be fostered by tumor-derived EVs. In this review, we will discuss the main body of literature describing how the cancer cells use the EVs for their growth into the bone and for educating the bone microenvironment to host metastases.

Keywords: bone tumor, extracellular vesicles, vicious cycle, dormancy, drug resistance, premetastatic niche

\section{EXTRACELLULAR VESICLES: AN INTRODUCTION}

\section{Biogenesis of EVs}

EVs are nanosized structures actively released by all cells (1). They are complex phospholipidic bilayers distinguishable in subtypes accordingly to their dimension: small EVs range below $100 \mathrm{~nm}$ (also known as exosome or exosome-like vesicles), middle/large EVs size between 100 and $1000 \mathrm{~nm}$ (also known as microvesicles and shedding vesicles), and finally apoptotic bodies, with a diameter ranging from 800 to $5000 \mathrm{~nm}$ (2). Nowadays apoptotic bodies are considered a stand-alone class of EVs, due to their peculiar biogenesis and biological functions, being mainly involved in programmed cell death. However, recent evidence has recognized an immunomodulatory role for these particles (1-3). Besides the size, the most important difference between the small and medium/large EVs lies in their biogenesis (4). In fact, small EVs arise from the fusion of endocytic 
vesicles sorted in a multivesicular body (MVB) via a complex molecular machinery involving endosomal sorting complex required for transport (ESCRT) components, ceramide/ sphingomyelinase pathway members, and Ras-related proteins in brain (RAB) proteins (4). This MVB then fuses with the plasma membrane and releases the small EVs in the extracellular space. Medium/large EVs arise directly from the plasma membrane via ADP-ribosylation factor (ARF) 6-mediated activation of phospholipase D (PLD), resulting in the recruitment of extracellular signal-regulated kinase (ERK) and phosphorylation of the myosin light-chain kinase (MLCK). This cascade triggers the budding of the cellular membrane, culminating in the formation and release of EVs from the cell (4). During their biogenesis, EVs entrap different macromolecules, such as nucleic acids (DNA, mRNA, miRNAs, long non-coding RNAs), lipids, and proteins (cytosolic factors, receptors, and ligands), which are then transferred to target cells where they induce metabolic changes (1).

\section{Paracrine and Systemic Effects of EVs}

Once EVs reach a target cell, the physical/molecular interactions between EV and cell membranes activate the EV uptake. This interaction has been shown to occur via multiple routes, including a direct fusion between EVs and the plasma membrane (5), as well as EV internalization via lipid draft-, clathrin-, and calveolae-dependent endocytosis, macropinocytosis, and phagocytosis (6-8). Indeed, the route of EVs uptake is likely dependent on the following factors: lipid and protein composition of the released EVs and of the plasma membrane of recipient cells, EV subtype, cell metabolic status, and extracellular space conditions (i.e. $\mathrm{pH}$, oxygen tension, and extracellular matrix components).

The contribution of EVs in the paracrine and distant communication is easily conceivable, since EVs can be found in all biological fluids and bloodstream and can reach every site of the body. However, to demonstrate these events is quite challenging. Lai et al. addressed this point by staining EVs with multiplexing bioluminescent (Gaussia luciferase, Gluc) and fluorescent (enhanced green fluorescent, EGFP, and tandem tomato, tdTomato) reporters into the mouse thymoma cell line EL4, finding that EV uptake and delivery of the mRNAs to recipient cells occurred within 1 hour (9). Furthermore, seeding EL4 cells in diffusion chambers, which are then subcutaneously implanted in the dorsal skin of mice, confirmed the systemic distribution of released EVs (9). Similar evidence was reported in another study, where EVs visualization and EVs cargo transfer between tumor cells and stromal cells in mice was assessed by high-resolution intravital imaging. Based on the Cre-LoxP system, the physiological effects of this exchange were studied in different melanoma and mammary tumor models (10). By generating Cre-expressing B16 melanoma cells and injecting them in mice expressing the Cre-LoxP reporter tdTomato, EVs release and uptake to distant organs (i.e., lymph nodes, lungs, and spleen) were observed within 2 weeks. In the same work, the authors showed that EVs released by $\mathrm{eGFP}^{+}$MDA-MB-231 breast cancer cells orthotopically injected in mice were taken up by the less malignant T47D tumor cells located into the contralateral mammary pad. Moreover, these EVs carry mRNAs involved in migration and metastasis. In fact, T74D cells that incorporated MDA-MB-231-EVs metastasized to lung 7.9-fold more than control cells (10). Later on, You and colleagues exploited the intrinsic optical properties of tissues and tumor-derived EVs based on their NADH content (11). In fact, they reached a labelfree visualization and characterization of metabolic fingerprint of EVs on a multiphoton microscopy via 2-photon fluorescence of $\mathrm{FAD}$, 3-photon fluorescence of $\mathrm{NAD}(\mathrm{P}) \mathrm{H}$, and third harmonic generation for the structural properties (lipid-water interface) of the EVs. With this method, EVs located inside fresh human breast biopsies collected from invasive ductal carcinoma patients were analyzed and compared to EVs collected from biopsies of healthy women subjected to breast reduction surgery, finding that the former presented with a higher $\mathrm{NAD}(\mathrm{P}) \mathrm{H}$ amount. Moreover, the concentration of NAD $(\mathrm{P}) \mathrm{H}$-rich EVs allowed not only to discriminate between patients and healthy subjects, but also to stratify the former according to tumor staging. However, this approach failed to reveal the dynamics of EVs in the tumor microenvironment, showing EVs firmly attached to the extracellular matrix and vessel walls, both in human biopsies and in animal models of breast tumor (11).

More recently, de Jong et al. described a CRISPR-Cas9-based reporter system to in vitro trace the EV-shuttling of small noncoding RNA molecules at single-cell resolution (12). They applied this technique on different breast cancer cell lines and demonstrated the functional transfer of small RNAs by means of EVs within 5 days. Moreover, knocking down genes involved in EV biogenesis and trafficking, such as Alix, Rab27a, Pak1, Rac1, Cav1, and RhoA, decreased EV uptake, while the silencing of Ankyrin Repeat And FYVE Domain Containing (ANKFY1), involved in vesicle transport, increased EV uptake (12).

\section{EXTRACELLULAR VESICLES IN BONE PHYSIOLOGY}

Bone is an active tissue in which different cell types live together and their crosstalk guarantees the mechanical, biochemical, and hormonal functions of the skeleton $(13,14)$. A fine cell-to-cell communication exists by means of membrane-bound and soluble molecules, allowing a selective spatial cellular differentiation and activity (15). The pivotal functional bone unit is the "bone remodeling unit" (BRU), constituted by bone forming osteoblasts and bone resorbing osteoclasts (16). Their activity is tightly coordinated by two main cytokines expressed by osteoblasts: macrophage-colony stimulating factor (M-CSF) and receptor activator of NF- $\mathrm{KB}$ Ligand (RANKL), having their receptor on pre-osteoclasts/monocytes: the colony stimulating factor 1 receptor (CSF1R, alias c-fms) and RANK, respectively (17). Once mature osteoclasts have resorbed the bone, a plethora of molecules are released from the extracellular matrix, affecting the osteoblast function and differentiation, such as transforming growth factor (TGF)- $\beta$, connective tissue growth factor (CTGF), 
osteocalcin (OCN), osteopontin (OPN), bone morphogenetic proteins (BMPs), insulin-like growth factor (IGF)-1 and -2 , platelet-derived growth factor (PDGF), and calcium ions (18, 19). Beside these molecules, many others concur to regulate this cellular crosstalk, such as stromal derived factor (SDF)-1, interleukin (IL)-1 and -6, fibroblast growth factor (FGF), parathyroid and parathyroid hormone related peptide (PTH/ PTHrP), Lipocalin-2 (LCN2), NOTCH family members, Ephrin ligands and receptors, Semaphorins, and vascular endothelial growth factors (VEGFs), that establish the so called "virtuous cycle of the bone" (20-23). In addition, the BRU establishes a further crosstalk with the bone/bone marrow-resident cells thorough juxtacrine and paracrine communications (24-26). Indeed, $\mathrm{EV}$ involvement in bone physiology has been elucidated in many aspects. The very first observation for a contribution of EVs in the process of bone mineralization was reported by two different groups in late ' 60 s $(27,28)$. Later on, EVs from osteoblasts were demonstrated to bind, by means of annexin 2, calcium phosphate, and other ions, thus triggering the formation of nuclei of mineralization in the bone matrix (29). Moreover, EVs have been described to be active coupling factors in osteoblastosteoclasts crosstalk. In fact, it is known that osteoblasts secrete EVs shuttling RANKL, which in turn sustains in vitro osteoclast formation (30). In line with this data, we found that osteoblastderived RANKL-positive EVs injected in osteoclast-poor RANKL knock out mice induced the osteoclastic commitment and the appearance of tartrate-resistant acid phosphatase (TRAP) positive cells in these mice (31). These results demonstrate another way exploited by osteoblasts to regulate osteoclast formation besides the juxtacrine and paracrine signaling. Vice versa, osteoblast differentiation and function can be also regulated by osteoclasts, as shown by Ma et al., who found that apoptotic bodies released by mature resorbing osteoclasts trigger osteoblast differentiation by activating the RANKL reverse signaling in these cells $(32,33)$. The pro-osteoblast differentiating effect could be also accomplished by an autocrine mechanism, since it has been demonstrated that EVs from mineralizing osteoblasts promote their own differentiation by activating Wnt- $\beta$-catenin pathway, while reducing Axin1 expression in bone marrow stromal cells (34). Recent reports show that osteocytes, the most abundant cells in bone having not only a mechanosensing role but also a regulatory role on osteoclast and osteoblast differentiation, accomplish these functions by releasing EVs. As a matter of fact, Morrel et al. found that under mechanical stress osteocytes release EVs, which in turn enhance in vivo bone formation (35). At the same time, osteocyte homeostasis is regulated by EVs, as demonstrated by Ren et al., who found that exosomes from adipose tissue MSCs counteracted the hypoxiaand serum deprivation-induced apoptosis of the osteocyte-like cell line MLO-Y4, eventually leading to a lower production of RANKL by these cells and, consequently, to an inhibition of osteoclast formation (36). Consistently, $\mathrm{Lu}$ and colleagues found that exosomes isolated from adipose tissue-derived MSCs promoted in vitro proliferation and osteogenic differentiation of primary human osteoblasts. Moreover, pre-conditioning of the donor cells with tumor necrosis factor (TNF)- $\alpha$ increased the pro-osteoblastogenic effect by a Wnt signaling dependent mechanism (37).
Finally, endothelial cells also contribute to bone homeostasis by releasing $\mathrm{EVs}$, which induce osteogenic differentiation of MSCs by shuttling Galectin-3 (38).

\section{EXTRACELLULAR VESICLES CONTRIBUTION TO TUMOR COLONIZATION OF BONE}

Neoplastic bone diseases are fueled by EVs (39). As a matter of fact, the onset and progression of both primary and metastatic bone cancers are promoted by a massive release of EVs, characterized by an abnormal molecular cargo (40). This event is not surprising considering that many key mechanisms involved in cancer progression, such as microenvironment acidification or aberrant pathways activation, also induce EVs production (41). In the bone context, tumor cells exploit the molecular pathways involved in bone remodeling to their own advantage, thus converting this "virtuous cycle" in a "vicious" one (42).

Different types of tumors can thrive in the bone milieu: primary tumors such as osteosarcoma (OS), Ewing's sarcoma, chondrosarcoma or fibrosarcoma and, very frequently, metastatic tumors from breast, prostate, lung, renal, colon, and bladder cancers $(43,44)$. All these tumors can hijack physiological stimuli to their advantage. Key examples are SDF-1, RANKL, and OPN, which can be exploited by cancer cells due to their chemoattractant and promitogenic activities, that summoning and promoting the engraftment of cancer cells into the bone microenvironment (45). The interaction between tumor cells and the bone induces a deregulation of bone giving rise to bone lesions can be classified as osteosclerotic, characterized by osteoblastic overactivation, osteolytic, due to an exacerbated osteoclast function, and mixed, when both features coexist in the same site $(46,47)$. Indeed, OS can generate all the three types of lesions, due to a wide histologic variety: the majority of OS cases ( $>85 \%)$ has osteosclerotic features, telangiectatic OS $(\sim 7 \%)$ presents osteolytic lesions, while small cell OS $(\sim 2 \%)$ produces mixed lesions $(48,49)$. In contrast, breast and lung cancers usually give rise to osteolytic bone metastases, while osteosclerotic lesions characterize most of prostate cancer-induced bone metastases (47-50).

\section{Extracellular Vesicles in Bone Tumors Growth}

$\mathrm{OS}$ is the most common primary tumor of bone, predominantly occurring in adolescents, with a second peak in elderly adults (51). MSCs and osteoblast precursors undergo malignant transformation, eventually leading to the deposition of an aberrant, immature bone (52). As for other types of tumors (Figure $\mathbf{1}$ and Table 1), growing evidence demonstrates a crucial impact of EVs on OS development. An interesting work by Macklin and colleagues demonstrated that the OS aggressive behavior can be transferred from the highly metastatic KHOS OS cell line to a non-metastatic KHOS subtype by means of EVs (67). This effect was due to an enrichment, inside the EVs, of molecules involved in G-protein coupled receptor signaling. Moreover, the highly metastatic OS cells secrete 3-fold more 


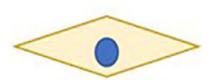

$\mathrm{CAFs} / \mathrm{MSCs}$

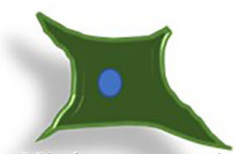

Higly-aggressive cancer cell

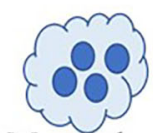

Macrophage
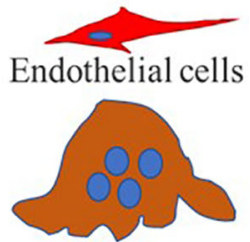

Osteoclast

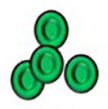

EVs

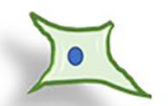

Bone cancer cell

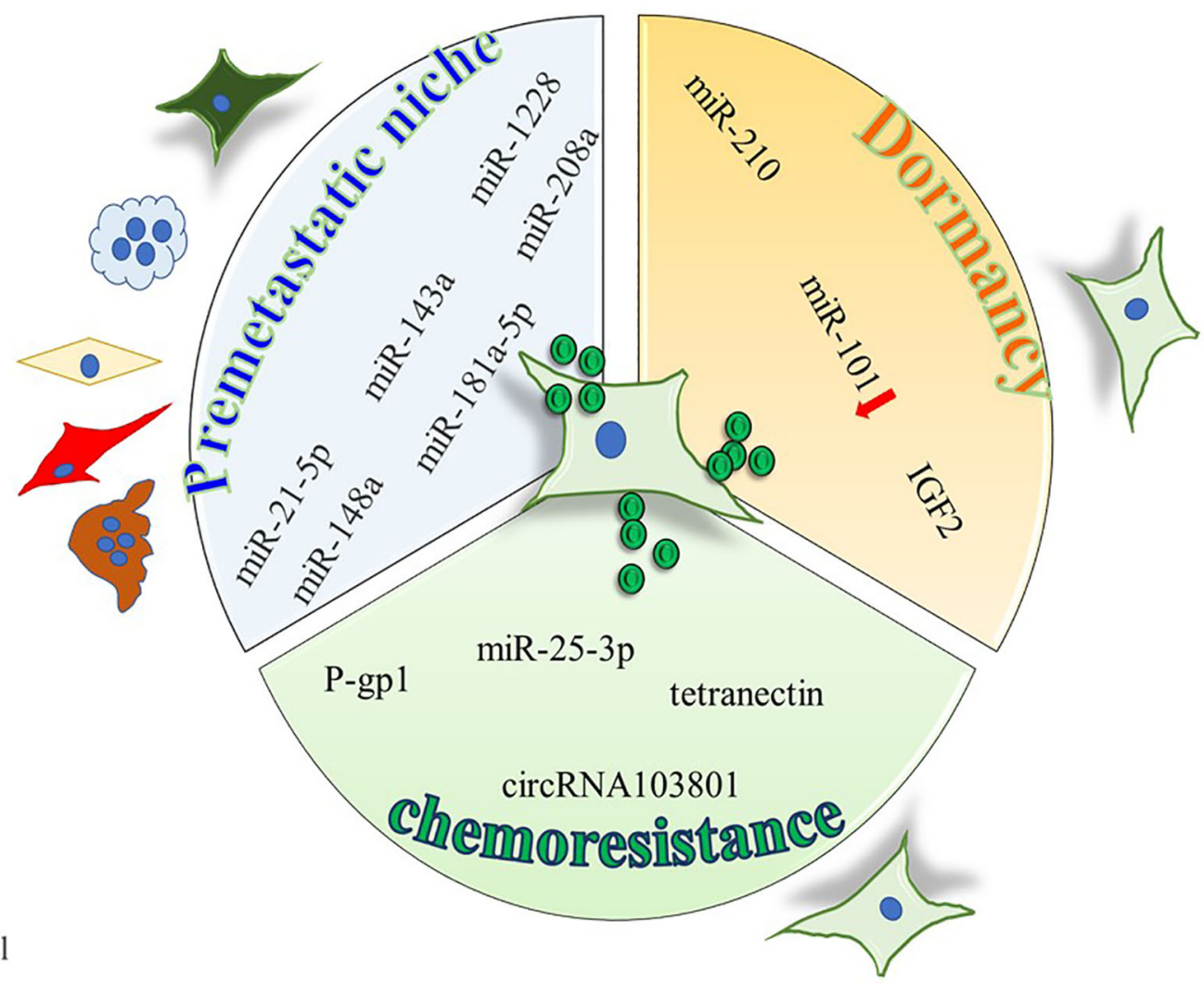

FIGURE 1 | Cartoon summarizing the intercellular molecular cargo exchanged by means of EVs in the context of primary bone tumors, between tumor cells and resident cells. Only molecules shuttled by EVs were indicated, all positively involved in premetastatic niche formation, tumor dormancy, and chemoresistance, except for miR-101, inversely correlated with dormancy potential.

TABLE 1 | Extracellular vesicles in the premetastatic niche in bone and in osteotropism provision.

\begin{tabular}{|c|c|c|c|c|}
\hline Donor & EV-Mediator & Target & Effect & Refs \\
\hline $\begin{array}{l}\text { Osteosarcoma cells U2-OS, } \\
\text { SAOS2, MG-63 }\end{array}$ & miR-148a, miR-21-5p & Osteoclasts,endothelial cells & $\begin{array}{c}\text { TRAP and MMP9 upregulation,VEGF, IL-6, -8 } \\
\text { upregulation }\end{array}$ & (53) \\
\hline SAOS2 & $\begin{array}{c}\operatorname{miR}-21-5 p, \operatorname{miR}-143-3 p, \operatorname{miR}-148 a-3 p \\
\text { and 181a-5p }\end{array}$ & MG63 & LOX, TIMP3 increase & (54) \\
\hline CAFs & $\operatorname{miR}-1228$ & MG-63 & SCAI suppression & (55) \\
\hline MSCs & miR-208a & SAOS2, MG63 & PDCD4 suppression & (56) \\
\hline Osteosarcoma LM7vcells & Whole EVs & Macrophages & Tgfb2, CCL2 & (57) \\
\hline 143-B, SAOS2 & Whole EVs & Alveolar macrophages & Immune cells recruitment & (58) \\
\hline Melanoma cells LCP & Whole EVs & $\begin{array}{c}\text { Melanoma cells WM-266, } \\
\text { SK-Mel28 }\end{array}$ & CXCR7 upregulaiton & (59) \\
\hline NSCLC, CRL-2868 lung cells & Amphiregulin & osteoclasts & EGFR induction & (60) \\
\hline Lung A549 & miR-192 & Endothelial cells & angiogenesis & (61) \\
\hline Breast cancer cells SCP28 & miR-21 & Osteoclasts & PDCD4 repression & (62) \\
\hline Prostate cancer cells C4 & miR-940 & Osteoblasts & ARHGAP1/FAM134A repression & (63) \\
\hline Prostate tumor cells TRAMP-C1 & Whole EVs & Osteoclast & Osteoclast differentiation decrease & (64) \\
\hline Prostate cancer cells PC-3 & Whole EVs & Osteoclasts & miR-214 and p-p65 downregulation & (65) \\
\hline $\begin{array}{l}\text { Prostate cancer cellsDu145 and } \\
\text { PC3 }\end{array}$ & TGF- $\beta$ & Adipose cells & VEGF-A, HGF, MMPs induction & (66) \\
\hline
\end{tabular}

vesicles than the lower metastatic ones, while multiphoton microscopy with fluorescence lifetime in vivo imaging demonstrated a preferential seeding of lungs by EVs derived from the highly metastatic OS clonal variants (67). Interestingly, Qi et al. showed that proliferation of the MG63 OS cell line is supported by EVs derived from bone marrow stromal cells, eventually leading to the activation of the Hedgehog pathway in the recipient cells (68), while other studies found that aggressiveness and metastatic 
potential of OS are directly related to the release of soluble and EVbound urokinase plasminogen activator (uPA) as well as EV-bound to PD-L1/N-cadherin $(69,70)$.

OS derived-EVs are also able to influence bone resident cells to promote OS grown. As an example, OS derived EVs stimulate endothelial cells to increase the secretion of IL-6,-8 and VEGF, by shuttling miR-148a and miR-21-5p (53). Osteoclasts are important players in OS, whose number seems to be correlated with tumor aggressiveness and metastatization (71). Interestingly, Garimella et al. found that EVs isolated from the 143B OS cell line contain a proosteoclastogenic cargo including matrix metalloproteinases- 1 and -13 (MMP-1, -13), TGF- $\beta$, CD-9, and RANKL, thus suggesting a role for these EVs in mediating bone degradation (72). Similarly, our recent work shows that EVs isolated from the MNNG/HOS human OS cell line significantly impair osteoblast differentiation while increasing osteoblast secretion of pro-osteoclastogenic/ inflammatory cytokines (i.e., IL-6, Lcn2, RankL, CCL2,5,6,12, and CXCL1,2,5) and of MMP3. Moreover, OS-derived EVs have a proangiogenic effect, evaluated both in vitro and in vivo (73).

Ewing's sarcoma is the second most common bone cancer in children and adolescents. It presents with small round cells derived from the neural crest and is usually associated with the chimeric fusion gene Ewing sarcoma breakpoint region 1/Friend leukemia integration 1 transcription factor (EWSR1/FLI1) (74). Miller et al. firstly described that Ewing's sarcoma-released EVs are enriched in the mRNA of the chimeric fusion gene, along with Six transmembrane epithelial antigen of the prostate 1 (STEAP1) and Lipase, member 1 (LIP1) mRNA (75). Interestingly, the specificity of this EV cargo could make circulating EVs suitable biomarker candidates for patients.

Chondrosarcoma accounts for approximately $20 \%$ of bone tumors and is due to a malignant transformation of chondroblasts (76). From an histological point of view, it is classified in three stages according to cytonuclear atypia, number of multinucleated cells, degeneration of chondroid matrix, and absence of mitosis. Also for this tumor, a contribution of EVs in its progression has been hypothesized. As an example, the SW1353 chondrosarcoma cellderived EVs shuttle the long non-coding RNA (lncRNA) RAMP2AS1, which acts as a molecular decoy for miR-2355-5p to regulate VEGFR2 expression thus increasing the angiogenic ability of HUVEC endothelial cells (77).

Finally, also the aggressiveness of fibrosarcoma seems to be supported by EVs release. This is a very rare bone tumor $(0.5$ cases in a million per year) caused by malignant transformation of fibroblasts (78). Malignant cells are strongly positive for vimentin and have an altered production of collagen, which is inversely correlated to the histological grade (78). Hakulinen et al. found that EVs isolated from the human fibrosarcoma cells HT-1080 contain both the inactive cleaved and the active full-length forms of MT1-MMP, which in turn activate pro-MMP2, allowing type I collagen and gelatin degradation (79).

\section{Extracellular Vesicles in Metastasis Development}

OS frequently metastasizes to lungs and its aggressiveness is related to osteoclastogenesis and neo-angiogenesis $(80,81)$. Likewise, it has been demonstrated that OS derived EVs increase both osteoclasts and neo-angiogenesis, by shuttling miR-148a and miR-21-5p to target cells (53). Jerez et al. investigated whether EV cargo can be predictive for metastatic potential of OS by investigating the miRNAs profile of EVs from metastatic SAOS2 and nonmetastatic MG63 osteosarcoma cell lines (82). They found that SAOS2-EVs were particularly enriched in miR-21-5p, miR-143-3p, miR-148a-3p, and 181a-5p compared to MG63-EVs. Consistently, bioinformatic analysis revealed, among the miRNA targets, some genes involved in ECM remodeling, such as the collagencrosslinking enzyme lysyl oxidase (LOX), and the tissue inhibitor metalloproteinase 3 (TIMP3), suggesting that SAOS2 related miRNAs may influence metastatic potential of OS at least in part by modulating ECM remodeling (82). Interestingly, another study highlights the protumoral role of cancer associated fibroblasts (CAFs) on OS by releasing EVs enriched in miR-1228. Once taken up by the OS cell lines MG-63 and HOS, miR-1228 suppressed SCAI (suppressor of cancer cell invasion), resulting in the promotion of migration and invasion of OS cells (83). Of note, MSCs have also been described to support osteosarcoma progression by releasing EVs (84). In particular, MSC-EVs enriched in miR-208a are taken up by SAOS2 and MG63 cells, which inhibited programmed cell death 4 (PDCD4) eventually stimulating tumor cells proliferation, migration and invasion (84).

Once reached the metastatic site, which is usually in the lungs, OS cells regulate the function of resident cells by means of EVs. Indeed, it has been reported that the LM7 metastatic osteosarcoma cell line releases EVs able to induce the production of IL-8, TGF$\beta 2$ and CCL22 from alveolar macrophages and the impairment of the macrophagic immune-surveillance function by promoting a switch of the M1-to-M2 phenotype (85). Interestingly, Mazumadar and colleagues found that EVs from the human OS cell lines 143B and SAOS2 (highly metastatic and non-metastatic, respectively) when injected intraperitoneally into SCID mice promote the recruitment of $\mathrm{CD} 11+\mathrm{Gr}+$ immune cells into lungs. Moreover, OS-derived EVs alone can recapitulate myeloid cell infiltration in the lungs of naïve mice but are insufficient to promote the development of OS metastasis, thus indicating that the establishment of the PMN in the lungs may require a combination of tumor-secreted factors along with EVs (86).

As already mentioned, to engraft and colonize a distant organ, cancer cells are able to influence the future host tissue by preparing the resident cells to receive tumor cells and support their growth (87). This process, generating a microenvironment suitable for cancer dissemination before the dissemination itself occurs, is called "premetastatic niche" (88). Many mechanisms and secreted factors inducing the premetastatic niche (PMN) have been discovered (89) and undoubtedly EVs are important players in this process. This is also true for bone metastases which, as already stated, represent the preferential secondary site of growth for several tumors. The accumulation of EVs in a metastatic site is accompanied by several modifications in the microenvironment, such as resident cells activation, matrix deposition and vascular proliferation (Figure 2 and Table 1).

Prostate cancer ( $\mathrm{PrCa}$ ) is the tumor with the highest propensity to metastasize to bone. In PrCa-induced bone 


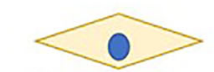

$\mathrm{CAFs} / \mathrm{MSCs}$

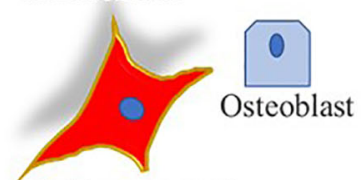

Highly metastatic cancer cell
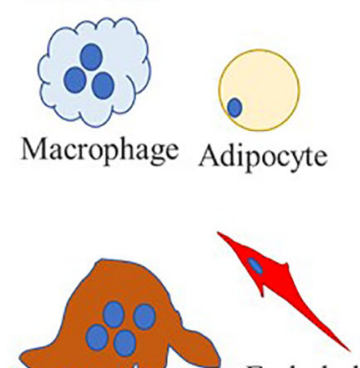

Endothelial

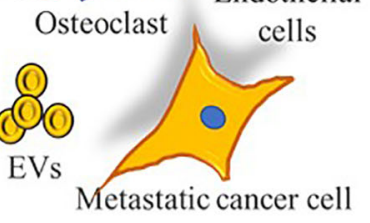

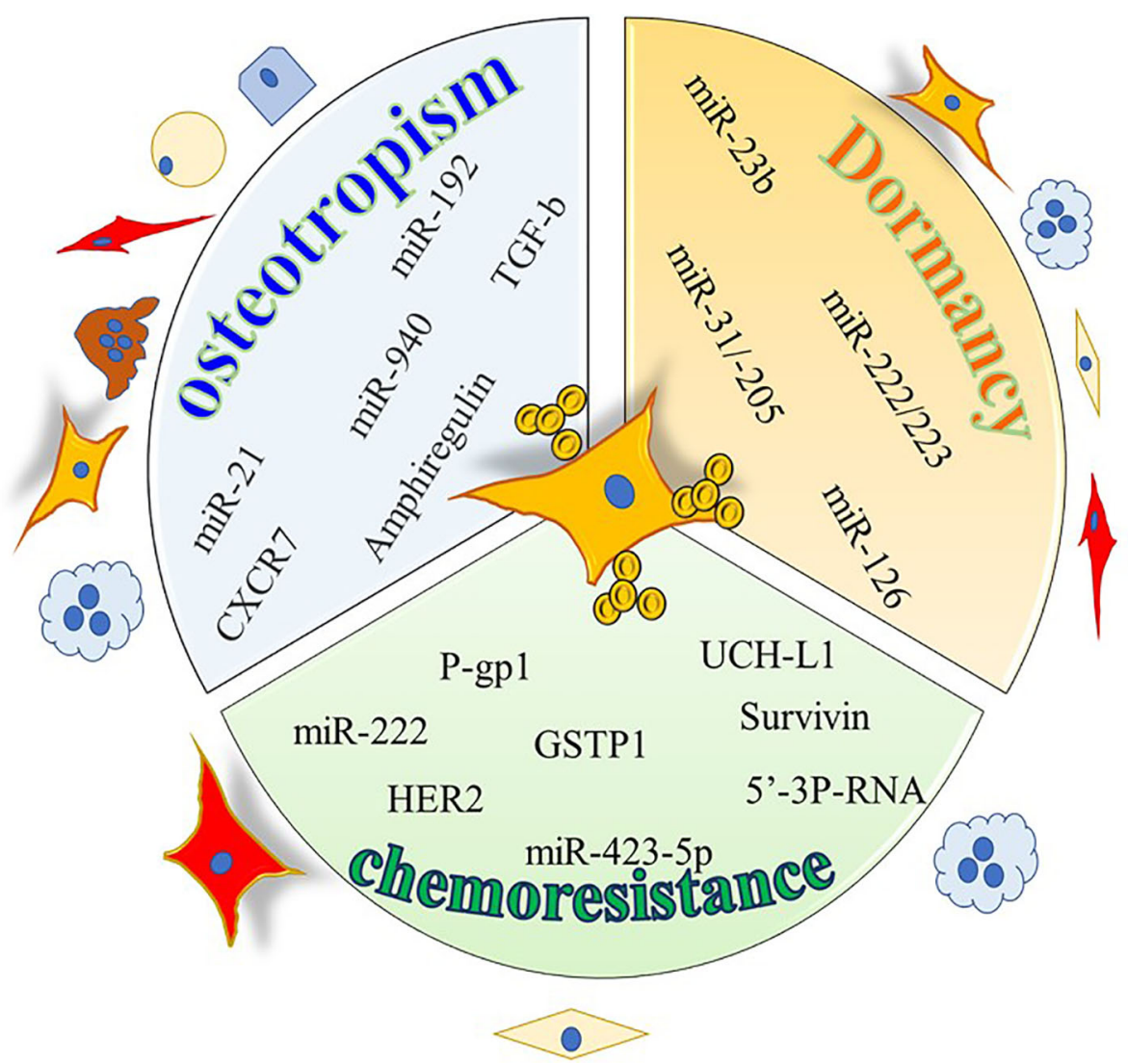

FIGURE 2 | Cartoon summarizing the intercellular molecular cargo exchanged by means of EVs between tumor cells and resident cells in bone metastases. Only molecules encompassed in EVs were indicated and reported to be positively involved in osteotropism, tumor dormancy, and chemoresistance.

metastases, several reports have emerged, showing the crucial role of tumor-derived EVs cargo in the preparation of the "soil". Hashimoto et al. found that miR-940 is highly expressed in EVs from highly osteotropic prostate cancer cells, and promotes osteoblastic/osteosclerotic bone lesions by targeting Rho GTPase Activating Protein 1 (ARHGAP1) and family with sequence similarity 134 member A (FAM134A) (90). Consistently, Karlsson et al. found that EVs from the TRAMP-C1 prostate tumor cell line impair osteoclast formation, while enhancing abnormal bone formation at the metastatic site (91). On the same line, other authors showed that PC3 PrCa cells decrease osteoclasts formation by means of EVs, which reduce the expression of miR-214 and the phosphorylation of p65 in osteoclast cells, finally suppressing the NF- $\mathrm{KB}$ signaling and osteoclast differentiation (92).These events enhance angiogenesis and finally tumor proliferation and invasiveness. PrCa cells also affect bone marrow MSCs behavior by means of EVs, by promoting a shift from an adipogenic toward a myofibroblastic differentiation. The latter cells in turn secrete high levels of proangiogenic (VEGF-A) and pro-invasive factors like MMP-1,-3 and -13 (93). Consistently PrCa cell (i.e., C4-2B, PC-3 and RWPE-1)derived EVs contribute to tumorigenic reprogramming of MSCs by delivering oncogenic transcripts of H-Ras, K-Ras and
oncomiRNAs miR-125b, miR-130b, and miR-155 as well as the Ras superfamily of GTPases Rabla, Rablb, and Rab1la (94). Finally, an autocrine effect of tumor cell-derived EVs was also demonstrated by Dai and Gao, who found that miR-183 shuttled by PC3 derived EVs promotes cancer cell proliferation, migration and invasion through the inhibition of Tropomyosin 1 (TPM1) (95).

With regards to breast cancer (BrCa), it also very frequently metastasizes to bone, inducing osteolytic lesions. EVs released by the SCP28 BrCa cells are enriched in miR-21 which is internalized by the osteoclasts (96). Inside these cells miR-21 represses PDCD4, a negative regulator of osteoclastogenesis, leading to an exacerbation of the osteoclast function. In agreement with these observations, breast cancer patients with bone metastasis showed higher expression of miR-21 in serum exosomes than those without metastasis or with non-bone metastasis (97).

Hypoxic BrCa cells 4T1 release miR-210-containing EVs in the tumor microenvironment resulting in inhibition of EphrinA3 and Protein tyrosine phosphatase 1B (PTP1B), as well as in the increase of angiogenesis and tumor proliferation (98). BrCaassociated fibroblasts secrete EVs that activate the Wnt-planar cell polarity (Wnt-PCP) and finally promote dissemination of 
cancer (99). Finally, we recently demonstrated that MDA-MB-231 derived-EVs affect bone cells in vitro, since they increase osteoclast formation while dramatically impairing osteoblast differentiation and activity. Moreover both in vitro (tube formation assay) and in vivo (Matrigel plug assay) experiments demonstrated the ability of these EVs to promote angiogenesis (100).

Melanoma is a very aggressive tumor, frequently generating visceral metastasis (101) while bone metastases occurr in around $15 \%$ of patients, with a dramatic worsening of quality of life and survival (102). An in vitro study from Mannavola and colleagues showed that the osteotropic melanoma LCP cells release EVs able to switch the non osteotropic WM-266 and SK-Mel28 cell lines to an osteotropic one, by increasing the expression of CXCR7 (103). In contrast, the expression of $\alpha_{6} \beta_{4}$ or $\alpha_{v} \beta_{5}$ integrins favors a specific affinity for the lung and the liver, respectively (54). In line with these observations, integrins $\beta_{4}$ and $\alpha_{v}$ expression in EVs isolated from the plasma of melanoma patients predict metastasis to lung and liver (54).

Lung cancer can also metastasize to the bone, and EVs facilitate this event (55). Indeed, EVs from the CRL-2868 non-small cell lung cancer (NSCLC) cell line and from lung cancer patients contain Amphiregulin (AREG) which in turn promotes osteoclast differentiation and exacerbated bone resorption by an EGFRdependent mechanism (56). In another study, metastatization to bone of lung adenocarcinoma A549 cells is reported to be inversely correlated miR-192 expression (57). In particular, the authors found that EVs from the highly metastatic A549-M1 subclone presented with a lower content of miR-192 compared to A549 parental EVs. Interestingly, intravenous administration of miR192-enriched EVs the day before the intracardiac injection of the A549-M1 subclone, drastically reduced the bone metastatic burden of these cells in the tibia, as well as the number of CD31 positive cells in comparison to naïve EVs (57).

\section{EXTRACELLULAR VESICLES CONTRIBUTION TO TUMOR CELL DORMANCY IN THE BONE}

Once cancer cells reach a distant organ, they can encounter a dormancy-permissive environment (58). In particular, the resident stem cell niche compartment can favors the survival of a distant cancer stem cell, by the same mechanisms usually employed to regulate the maintenance of the stem cell pool and the recruitment of uncommitted cells into the tissue (59, 104, 105). On the other side, the dormant tumor cells can acquire stem cell-like properties overexpressing specific genes to interact with the niche (46). As an example, BrCa cells can shift toward a dormant phenotype in the endosteal niche, activating a hematopoietic stem cell profile via the Notch2 pathway (106). Cancer dormancy accounts for a considerable clinical problem, since it is responsible for tumor recurrence; in fact the tumor awakening leads to the generation of secondary tumor lesions, which in many cases constitute the cause of the death of patients (107).

Several studies revealed that EVs can also trigger the dormant phenotype of tumor cells (Figure 1 and Table 2). Kling et al. found that under hypoxic conditions, the Ewing's sarcoma cells A673 and SK-ES-1 upregulate and load miR-210 in their EVs, which suppresses Caspase 8 Associated Protein 2 (CASP8AP2) expression in parental cells, eventually inhibiting OS cells apoptosis and inducing a stem-like phenotype (60). Furthermore, OS modulate its aggressiveness and dormant phenotype by the EVshuttled miR-101 (61). In fact, in vivo injection of SAOS2 and SOSP-9607 cells overexpressing miR-101 resulted in less lung metastases in mice, through the silencing of B cell lymphoma protein, BCL6. Consistently, when miR-101 was overexpressed in MSCs, the MSC-EVs were enriched in this miRNA and effectively reduced in vitro migration and invasion of SAOS2 and 143B cells. These effects have been confirmed in immunocompromised mice, in which the treatment with MSC-EVs-miR-101 reduced the lung metastatic foci after 2 weeks of intratibial injection of 143B. In addition, other studies found that plasma EV-miR-101 is able to distinguish between OS patients with metastasis from those without. Insulin like growth factor (IGF)2, was reported to be shuttled by EVs and was isolated from biological fluids of healthy subjects as well as of cancer patients (62). Moreover, OS survival is sustained by IGF2, that induced dormancy by triggering autophagic pathways and inducing chemoresistance to adriamycin in syngenic animal models injected with the OS murine ATX cells (117). Notably, in biopsies from OS patients with favorable prognosis after chemotherapy, IGF2 levels are decreased.

With regards to bone metastases (Figure 2 and Table 2), in an in vitro study Ono et al. found that EVs from MSCs decrease the proliferation, invasion, and sensitivity to chemotherapeutics of the bone metastatic human BrCa BM2 cells (63). These effects were due to the shuttling of miR-23b from MSC-EVs to cancer cells,

TABLE 2 | Extracellular vesicles in the support of tumor dormancy in the bone.

\begin{tabular}{|c|c|c|c|c|}
\hline Donor & EV-Mediator & Target & Effect & Refs \\
\hline EWS cells A673,SK-ES-1 & miR-210 & Autologous & CASP8AP2 silencing & (108) \\
\hline SAOS2, SOSP-9607, 143-B & miR-101 & Autologous & BCL6 silencing & (109) \\
\hline AXT osteosarcoma cells & IGF2 & Autologous & Autophagy induction & $(110)$ \\
\hline MSCs & miR-23b & Breast cancer cells BM2 & MARKS suppression & $(111)$ \\
\hline Breast cancer cells MDA-MB-231, T47D & $\mathrm{miR}-222 / 223$ & MSCs/autologous & Switch in G1-G0 phase & $(112)$ \\
\hline Breast cancer cells MDA-MB-231, T47D & Whole EVs & M1/M2 macrophages & Cancer proliferation/dormancy & $(113)$ \\
\hline MSCs & miR-31/-205 & Breast cancer cells MDA-MB-321 & UBE2N suppression & $(114)$ \\
\hline MSCs & Whole EVs & Breast cancer cells MCF7 & Dormancy induction & $(115)$ \\
\hline Endothelial cells & miR-126 & Leukemic cells & Dormancy induction & $(116)$ \\
\hline
\end{tabular}


which suppresses MARKS (myristoylated alanine-rich C kinase substrate) expression, encoding for a protein that promotes cell cycling and motility. Likewise, a study from Bliss demonstrated that BrCa cells promote the MSCs release of EVs enriched in miR$222 / 223$, which in turn arrested into G1-G0 phase a subset of cancer cells, thus favoring a quiescent phenotype. In contrast, naïve MSC-derived EVs induced MDA-MB-231 cells into cycling (64). The same group dissected the crosstalk of the bone marrow macrophages with dormant $\mathrm{BrCa}$ cells, finding that M2 macrophages support the dormancy of $\mathrm{BrCa}$ cells through gap junctions, arresting tumor cell cycle. Interestingly, Walker et al. demonstrated that M1 macrophages release EVs able to awake cancer cells and induce proliferation, migration, and epithelial to mesenchymal transition (65). Another study revealed that MSCs support the dormancy of MDA-MB-321 through the EVmediated shuttling of miR-31 and 205 (66). These miRNAs target ubiquitin conjugating enzyme E2 $N$ (UBE2N) gene, suppressing the proliferation, migration, and invasion of tumor cells in bone. Similarly, proliferation and migration ability were reduced in MCF7 BrCa cells treated with MSC-EVs while enhancing cell adhesion (118).

\section{EXTRACELLULAR VESICLES IN BONE CANCER DRUG RESISTANCE}

Overcoming chemoresistance is a major clinical unmet need, since what is usually the first line treatment is not an option for patient with chemoresistant cancer. Many mechanisms have been elucidated in the establishment of chemoresistance, and EVs have been shown to shuttle the necessary molecular machinery (Figures 1, 2 and Table 3) to promote drug resistance (131).

\section{Drug Resistance in Bone Tumors: Role of EVs}

Primary OS MG63 cells treated with doxorubicin (DXR) increased their expression of P-glycoprotein (P-gp)1, a membrane transporter pumping xenobiotics outside the cell. Consistently, EVs from DXR treated MG63 presented with higher levels of both $A B C B 1$ transcript and P-gp encoded pretein expression, that can be transferred to untreated MG63 cells, conferring them drug resistance (132). In another study, upregulation of miR-25-3p, which silences the Dickkopf WNT signaling pathway inhibitor 3 (DKK3) gene, was detected in human OS samples, where it is negatively correlated with clinical outcome (133). The same authors demonstrated that miR-25$3 \mathrm{p}$ upregulation supported tumor growth and drug resistance and that the same effects were observed after DKK3 silencing. Interestingly, miR-25-3p was found in cell-derived EVs. In another study by Weinman et al. the correlation between drug resistance and EVs has been investigated in a spontaneous canine model of OS (134). The authors clustered the animals in two cohorts based on the responsiveness to amputation and adjuvant carboplatin chemotherapy (good, disease-free interval > 300 days; poor, diseasefree interval $<100$ days) and analyzed the protein profile of circulating EVs by mass spectrometry. The proteomic profile revealed differences in EV cargo, identifying tetranectin, which was decreased in the poor prognosis group, as the most reliable biomarker.

The clinical relevance of EV-mediated drug resistance in OS patients has been investigated by Pan et al. (135). The analysis of circulating EVs from 43 OS patients compared to healthy subjects revealed overexpression of the circular RNA circRNA103801 in the former. The level of this circRNA presented a prognostic value, being inversely correlated with the overall survival of patients. The authors further investigated the biological function of the circRNA103801 in an in vitro model of OS. The overexpression of circRNA103801 in MG63 cells increased the sensitivity to cisplatin, the OS cells released also EV enriched in the circRNA. When these EVs were incubated to naïve MG63 and U2OS cells, both the OS cells increased the sensitivity to cisplatin, upregulating the expression of P-gp and Multidrug Resistance Protein 1, MRP1. These experiments demonstrated that circRNA103801 is responsible for conferring chemoresistance in OS patients.

\section{Drug Resistance in Bone Metastases: Role of EVs}

Like in OS, the expression P-gp was increased in MCF7 BrCa cells after exposure to docetaxel (DOC) Moreover, EVs from

TABLE 3 | Extracellular vesicles in cancer drug-resistance in the bone.

\begin{tabular}{|c|c|c|c|c|}
\hline Donor & EV-Mediator & Target & Effect & Refs \\
\hline MG-63 & P-gp1 & Autologous & Resistance to doxorubicin & (119) \\
\hline Osteosarcoma biopsies, 143B, U2-OS & miR-25-3-p & Autologous & Dkk3 silencing & (120) \\
\hline Osteosarcoma HMPOS cells, canine osteosarcoma biopsies & Tetranectin & Autologous & Resistance to cisplatin & $(121)$ \\
\hline Seric EVs from patients,MG63, U2OS cells & circRNA103801 & Autologous & Resistance to cisplatin & \\
\hline Breast cancer cells MCF7 & P-gp1 & Autologous & Resistance to docetaxel & (122) \\
\hline Breast cancer cells MCF7 & $\mathrm{UCH}-\mathrm{L} 1$ & Autologous & Resistance to doxorubicin & (123) \\
\hline Breast cancers cells MCF7 & miR-222 & M2 macrophages & PTEN silencing and $\mathrm{M} 2$ proliferation & (124) \\
\hline Breast cancer cells MCF7 & miR-222 & Autologous & Resistance to tamoxifen & (125) \\
\hline Breast cancer cells MCF7 & GSTP1 & Autologous & Resistance to doxorubicin & (126) \\
\hline MDA-MB-231 & Survivin & Autologous & Resistance to paclitaxel & (127) \\
\hline Breast cancer cells SKBR3 and BT474 & HER2 & Autologous & Resistance to trastuzumab & (128) \\
\hline CAFs & $\begin{array}{c}\text { 5'-triphospate } \\
\text { RNA }\end{array}$ & Breast cancer cells & Radiation- and chemo-resistance & (129) \\
\hline CAFs & miR-423-5p & prostate cancer cells(LNCAP, 22RV-1, C4) & Resistance to taxane & (130) \\
\hline
\end{tabular}


DOC-treated MCF-7 expressed higher levels of P-gp compared to EVs from naïve MCF-7, and the incubation with DOC-MCF-7 EVs reduced cell apoptosis of naïve MCF-7 (108). It is known that $A B C B 1$ can be activated by the ubiquitin carboxy-terminal hydrolase (UCH) L1 through MAPK/ERK pathway. Consistently, MCF7 treated with adriamycin (ADR) show high level of UCH-L1 and phospho-ERK compared to control cells and when naïve MCF7 cells were cultured with EVs from ADR-MCF7 they acquired a reduced sensitivity to ADR and increased p-ERK and P-gp levels (109). Interestingly, EVs from blood of BrCa patients were positive for UCH-L1 and show an inverse correlation with chemosensitivity.

Chemotherapeutics resistance can also occur via EV-mediated miRNAs transfer. In fact, $\mathrm{ADR} / \mathrm{DOC}$-resistant $\mathrm{BrCa}$ cells release EVs enriched in miR-222, suppressing phosphatase and tensin homolog (PTEN) gene, a tumor suppressor which negatively regulates intracellular levels of phosphatidylinositol trisphosphate and the Akt signaling (136). These EVs are also taken up by M2 macrophages, eventually promoting their activation and polarization to support cancer cells. Accordingly, miR-222 has been also found in EVs from plasma and tissue of chemoresistant patients (110). Exosomal miR-222 is also responsible for the resistance to tamoxifen in MCF7 cells, suppressing p27 and Estrogen Receptor (ER) alpha expression (110).

Cancer cells can counteract chemotherapeutics like ADR by overexpressing the glutathione-S-transferase P1 (GSTP1), a phase II-metabolizing enzyme that detoxifies chemicals by conjugating with glutathione. Yang et al. found that this enzyme is present in EVs from ADR/MCF7 and sera of chemoresistant patients (111). Another study described that, when exposed to paclitaxel (PTX), MDA-MB-231 specifically released EVs enriched in Survivin, an inhibitor of apoptosis (112). The protective effects of Survivinenriched EVs were effective on drug-sensitive fibroblasts and SKBR3 cells when exposed to PTX. Furthermore, tumor cells can directly counteract chemotherapeutics by EV release, via a decoy-like system. Indeed, Ciravolo et al. described that the human epidermal growth factor receptor 2 (HER2)-positive BrCa cells SKBR3 and BT474 release HER2-positive EVs. These EVs are able to bind and neutralize the biological drug trastuzumab, an anti-HER2 antibody, while EVs from triple negative BrCa cells MDA-MD-231 do not (113). In fact, SKBR3 cells treated with autologous EVs were less sensitive to the antiproliferative effect of trastuzumab. Interestingly, the authors found that EVs purified from sera of HER2-positive BrCa patients showed lower binding to trastuzumab compared to EVs circulating in sera from patients with advanced disease.

Notably, cancer cells can also take advantage of CAFs derived EVs to acquire chemoresistance. Indeed, it has been shown that EVs from CAFs sustain radiation- and chemo-resistance of MDA-MB-231 BrCa cells by activating retinoic acid-inducible gene I (RIG-I), signal transducer and activator of transcription (STAT) 1, and NOTCH3 pathways (114). Similarly, Shan et al. dissected the role of CAF-EVs in taxane resistance-acquisition by PrCa cells, finding an enrichment of miR-423-5p in these EVs which, once internalized in PrCa cells (LNCAP, 22RV-1 and C4 cells) suppressed GREM2 (encoding for gremlin2 protein inhibitor of bone morphogenetic proteins family members,
BMPs) and increased TGF- $\beta$ eventually leading to a reduced sensitivity to taxane (115).

\section{POTENTIAL APPLICATIONS OF EVS IN CLINICAL MANAGEMENT}

Cancer-derived EVs can be also used for diagnosis and to monitor cancer progression, exploiting them in the field of liquid biopsy. Of note, fibronectin shuttled by EVs isolated from $\mathrm{BrCa}$ patients is becoming a reliable diagnostic marker for detecting tumor early stages (116). On the same line, developmental endothelial locus-1 protein (Del-1) has been proposed as an exosomal biomarker to discriminate between benign and malignant $\mathrm{BrCa}$ (119). Periostin is another potential EV-related biomarker, since it has been found enriched in circulating exosomes from $\mathrm{BrCa}$ patients, and allowing the stratification of patients with localized disease versus those with lymph node metastasis (120). HER2 expression in circulating EVs has been found to be consistent with the positivity assessed by immunohistochemistry on tumor biopsies (121). Similarly, PrCa diagnosis can be addressed via EVs, since the classical marker prostate specific antigen (PSA) can be measured in EVs from patients, distinguishing benign hyperplasia from malignant transformation (137). EV-bound survivin has been proposed as a biomarker and clinical tool for diagnosing or monitoring PrCa as well as PTEN, both being detectable only in patients (138). Melanoma can be also monitored by circulating EVs profile of caveolin 1, and lung cancers can be histologically discriminated through a multimarker model based on the expression on circulating EVS of tetraspanin 8/CD151/CD171 $(122,139)$. Not only protein cargoes but also miRNA profiles of circulating EVs in patients suffering from cancers are pursued for prognostic and diagnostic aims (123-126).

Another useful approach in the EVs field is their employment as pharmacological delivery system for the treatment of different cancers (127). Indeed, EVs present with attractive potentialities due to a relatively long half-life, high biocompatibility, and minimal or no adverse effects. As an example, Melzer et al. showed the efficacy of taxol-loaded MSC-derived exosomes in targeting MDA-MB-231 growth and metastases in NOD-SCID mice (128). Similarly, zoledronate and dasatinib were efficaciously delivered by osteoblast-EVs and suppressed osteoclast formation and function in vivo, thus potentially being efficacious in reducing tumor-induced osteolysis (31). Consistently, EVs from the monocyte/macrophage cell line RAW264.7 have been reported to be efficient vehicles for paclitaxel and doxorubicin shuttling and treatment of mice orthotopically injected with MDA-MB-231 cells (129).

Finally, EVs are promising tools for immunotherapy (130). As an example, EVs derived from overexpressing IL-12 transgenic renal and bladder cancer cells efficiently trigger a strong antitumoral activity of cytotoxic T cells through the FasL/ Fas signaling pathway, and the same strategy could be applied to bone primary tumors (140). Xu's group demonstrated that EVs 
from macrophages carry phagocytosed antigens to dendritic cells and strengthen $\mathrm{T}$-cell responses, conferring anti-tumor immunity (141). Dendritic cell-derived EVs induced specific cytotoxic $\mathrm{T}$ cell activation suppressing the growth of lung adenocarcinoma in a xenograft mouse model (142), while Zhu et al. found that NK cell primed by IL-15 shuttled-EVs show a higher cytolytic effect against BrCa cells MDA-MB-231 injected in nude mice (143). Many other studies and ongoing clinical trials are endorsing these approaches.

\section{OUTLOOK AND PERSPECTIVES}

The whole body of evidence from the literature strongly indicates that EVs exert a critical role in the progression of both primary and secondary bone tumors. Bone is an attractive microenvironment for tumor cells growth and metastasis, through the establishment of a crosstalk among many different resident cell types, occurring via juxtacrine, paracrine, and EV-mediated mechanisms. The latter are an additional piece of the complex system by which tumor fuels this "vicious cycle". The translational significance of this aspect is endorsed by the growing interest of the application of EVs in diagnostic and therapeutic fields, as well as the patent applications/grants and clinical trials based on the use of EVs. However, many challenges are still present in the field. For the basic research side the main issues are: i) to isolate with very high purity the different EV subpopulations, ii) to identify specific mechanisms of selective EV uptake into target cells, iii) to track the kinetics and distribution of EVs towards distant tissues, and iv) to distinguish the real contribution of the molecular legacy of EVs into an complex microenvironment, such as the tumor milieu. The isolation and quantification methods are still a crucial issue. Depending of the technique used for the isolation, the composition and the effect of the EVs from the same source can be different, and the same goes for the quantification. Of course, there are consensus methods in the field, but every method presents pitfalls to take into account. Protein titration, one of the most widely used methods in laboratory practice, can be affected by "exogenous" contaminants and does not consider the size distribution. Methods of quantification based on physical features, such as dynamic light scattering and resistive pulse sensing, cannot estimate the entire EV population, since generally a size cut-off must be applied on the instruments, and cannot distinguish between intact and damaged EVs.

The possible experimental approaches to dissect biological aspects of EVs have pros and cons: the in vitro studies can help

\section{REFERENCES}

1. Raposo G, Stahl PD. Extracellular Vesicles: A New Communication Paradigm? Nat Rev Mol Cell Biol (2019) 20(9):509-10. doi: 10.1038/ s41580-019-0158-7

2. Théry C, Witwer KW, Aikawa E, Alcaraz MJ, Anderson JD, Andriantsitohaina R, et al. Minimal Information for Studies of Extracellular Vesicles 2018 (MISEV2018): A Position Statement of the International Society for Extracellular Vesicles and Update of the MISEV2014 Guidelines. J Extracell Vesicles (2018) 7:1535750. doi: 10.1080/20013078.2018.1535750 to quantify and estimate the contribution of a specific pathway in the cellular context. However, the in vitro cell system cannot take in consideration the paracrine and systemic contribution of all cell types on cancer metabolism and related EVs. Furthermore, the EV cargo is a balanced cocktail of molecules that can exert opposite effects (i.e., EVs can shuttle both RANKL and OPG), and generally the investigators focus only on one member of them, possibly overestimating the importance of the single factor using targeted rather than system/pathway-oriented overexpression or knockdown techniques. On the other side, the in vivo studies in animal models can help to assess the real contribution of a molecular player on a biological system, but many confounding factors are present due to the complexity of the system. The in vivo studies help to investigate the kinetics of release and integration of EVs, frequently using intravital microscopy approaches. These studies provide impactful data, but are limited by technical constraints, such as resolution, signal/noise ratio and cellular recycling of the tags used for tracking the EVs.

Finally, a real clinical setting of EVs is hindered by many limitations. The main technical issues are shared with basic research. Indeed, a lack of standardization methods for isolation, characterization, quality control, large scale production and storage conditions reduce the translational value of EVs (144). Moreover, the EVs and their molecular profile can change for several patient-related variables, including the circadian rhythm and lifestyle habits as well as comorbidities, affecting their prognostic reliability $(145,146)$.

In conclusion, EVs are an active field of study for a better understanding of the biological bases of tumorigenesis, but also offer a promising translational tool for diagnosis, monitoring, and treatment of cancer patients.

\section{AUTHOR CONTRIBUTIONS}

AC and NR contributed to original draft conceptualization, preparation, and revision. Both authors have read and agreed to the final version of the manuscript. All authors contributed to the article and approved the submitted version.

\section{FUNDING}

“Associazione Italiana per la Ricerca sul Cancro" (AIRC, \#IG 2020 ID 24823) to NR.
3. Caruso S, Poon IKH. Apoptotic Cell-Derived Extracellular Vesicles: More Than Just Debris. Front Immunol (2018) 9:1486. doi: 10.3389/fimmu.2018.01486

4. Colombo M, Raposo G, Théry C. Biogenesis, Secretion, and Intercellular Interactions of Exosomes and Other Extracellular Vesicles. Annu Rev Cell Dev Biol (2014) 30:255-89. doi: 10.1146/annurev-cellbio-101512-122326

5. Parolini I, Federici C, Raggi C, Lugini L, Palleschi S, De Milito A, et al. Microenvironmental $\mathrm{pH}$ is a Key Factor for Exosome Traffic in Tumor Cells. J Biol Chem (2009) 284:34211-22. doi: 10.1074/jbc.M109.041152

6. Nanbo A, Kawanishi, Yoshida R, Yoshiyama H. Exosomes Derived From Epstein-Barr Virus-Infected Cells are Internalized via Caveola-Dependent 
Endocytosis and Promote Phenotypic Modulation in Target Cells. J Virol (2013) 87:10334-47. doi: 10.1128/JVI.01310-13

7. Fitzner D, Schnaars M, van Rossum D, Krishnamoorthy G, Dibaj, Bakhti M, et al. Selective Transfer of Exosomes From Oligodendrocytes to Microglia by Macropinocytosis. J Cell Sci (2011) 124:447-58. doi: 10.1242/JCS.074088

8. Feng D, Zhao WL, Ye YY, Bai XC, Liu RQ, Chang LF, et al. Cellular Internalization of Exosomes Occurs Through Phagocytosis. Traffic (2010) 11:675-87. doi: 10.1111/J.1600-0854.2010.01041.X

9. Lai CP, Kim EY, Badr CE, Weissleder R, Mempel TR, Tannous BA. Breakefield XO.Visualization and Tracking of Tumour Extracellular Vesicle Delivery and RNA Translation Using Multiplexed Reporters. Nat Commun (2015) 6:7029-41. doi: 10.1038/NCOMMS8029

10. Zomer A, Maynard C, Verweij FJ, Kamermans A, Schäfer R, Beerling E, et al. In Vivo Imaging Reveals Extracellular Vesicle-Mediated Phenocopying of Metastatic Behavior. Cell (2015) 161:1046-57. doi: 10.1016/j.cell.2015.04.042

11. You S, Tu H, Chaney EJ, Sun Y, Zhao Y, Bower AJ, et al. Intravital Imaging by Simultaneous Label-Free Autofluorescence-Multiharmonic Microscopy. Nat Commun (2018) 9:1-9. doi: 10.1038/s41467-018-04470-8

12. De Jong OG, Murphy DE, Mäger I, Willms E, Garcia-Guerra A, GitzFrancois JJ, et al. A CRISPR-Cas9-Based Reporter System for Single-Cell Detection of Extracellular Vesicle-Mediated Functional Transfer of RNA. Nat Commun (2020) 11:1-13. doi: 10.1038/s41467-020-14977-8

13. Datta HK, Ng WF, Walker JA, Tuck SP, Varanasi SS. The Cell Biology of Bone Metabolism. J Clin Pathol (2008) 61:577-87. doi: 10.1136/jcp.2007.048868

14. Cappariello A, Ponzetti M, Rucci N. The "Soft" Side of the Bone: Unveiling its Endocrine Functions. Horm Mol Biol Clin Investig (2016) 28:5-20. doi: 10.1515/hmbci-2016-0009

15. Matsuo K, Irie N. Osteoclast-osteoblast Communication. Arch Biochem Biophys (2008) 473:201-9. doi: 10.1016/j.abb.2008.03.027

16. Sims NA, Martin TJ. Coupling the Activities of Bone Formation and Resorption: A Multitude of Signals Within the Basic Multicellular Unit. Bonekey Rep (2014) 3:481-91. doi: 10.1038/bonekey.2013.215

17. Cappariello A, Maurizi A, Veeriah V, Teti A. The Great Beauty of the Osteoclast. Arch Biochem Biophys (2014) 561:13-21. doi: 10.1016/j.abb.2014.08.009

18. Sims NA, Martin TJ. Osteoclasts Provide Coupling Signals to Osteoblast Lineage Cells Through Multiple Mechanisms. Annu Rev Physiol (2020) 82:507-29. doi: 10.1146/annurev-physiol-021119-034425

19. Zayzafoon M. Calcium/calmodulin Signaling Controls Osteoblast Growth and Differentiation. J Cell Biochem (2006) 97:56-70. doi: 10.1002/jcb.20675

20. Rucci N, Capulli M, Piperni SG, Cappariello A, Lau P, Frings-Meuthen P, et al. Lipocalin 2: A New Mechanoresponding Gene Regulating Bone Homeostasis. J Bone Miner Res (2015) 30:357-68. doi: 10.1002/jbmr.2341

21. Peruzzi B, Cappariello A, Del Fattore A, Rucci N, De Benedetti F, Teti A. CSrc and IL-6 Inhibit Osteoblast Differentiation and Integrate IGFBP5 Signalling. Nat Commun (2012) 3:630. doi: 10.1038/ncomms1651

22. Mundy GR, Elefteriou F. Boning Up on Ephrin Signaling. Cell (2006) 126:441-3. doi: 10.1016/j.cell.2006.07.015

23. Verlinden L, Vanderschueren D, Verstuyf A. Semaphorin Signaling in Bone. Mol Cell Endocrinol (2016) 432:66-74. doi: 10.1016/j.mce.2015.09.009

24. Han Y, You X, Xing W, Zhang Z, Zou W. Paracrine and Endocrine Actions of Bone - The Functions of Secretory Proteins From Osteoblasts, Osteocytes, and Osteoclasts. Bone Res (2018) 6:1-11. doi: 10.1038/s41413-018-0019-6

25. Kusumbe AP, Ramasamy SK, Adams RH. Coupling of Angiogenesis and Osteogenesis by a Specific Vessel Subtype in Bone. Nature (2014) 507:323-8. doi: 10.1038/nature13145

26. Ponzetti M, Rucci N. Updates on Osteoimmunology: What's New on the Cross-Talk Between Bone and Immune System. Front Endocrinol (Lausanne) (2019) 10:236. doi: 10.3389/fendo.2019.00236

27. Anderson HC. Electron Microscopic Studies of Induced Cartilage Development and Calcification. J Cell Biol (1967) 35:81-101. doi: 10.1083/jcb.35.1.81

28. Bonucci E. Fine Structure of Early Cartilage Calcification. J Ultrastruct Res (1967) 20:33-50. doi: 10.1016/S0022-5320(67)80034-0

29. Davies OG, Cox SC, Williams RL, Tsaroucha D, Dorrepaal RM, Lewis MP, et al. Annexin-Enriched Osteoblast-Derived Vesicles Act as an Extracellular Site of Mineral Nucleation Within Developing Stem Cell Cultures. Sci Rep (2017) 7:12639. doi: 10.1038/s41598-017-13027-6

30. Deng L, Wang Y, Peng Y, Wu Y, Ding Y, Jiang Y, et al. Osteoblast-Derived Microvesicles: A Novel Mechanism for Communication Between
Osteoblasts and Osteoclasts. Bone (2015) 79:37-42. doi: 10.1016/ j.bone.2015.05.022

31. Cappariello A, Loftus A, Muraca M, Maurizi A, Rucci N, Teti A. OsteoblastDerived Extracellular Vesicles are Biological Tools for the Delivery of Active Molecules to Bone. J Bone Miner Res (2018) 33:517-33. doi: 10.1002/ jbmr.3332

32. Ma Q, Liang M, Wu Y, Ding N, Duan L, Yu T, et al. Mature OsteoclastDerived Apoptotic Bodies Promote Osteogenic Differentiation via RANKLMediated Reverse Signaling. J Biol Chem (2019) 294:11240-7. doi: 10.1074/ jbc.RA119.007625

33. Ikebuchi Y, Aoki S, Honma M, Hayashi M, Sugamori Y, Khan M, et al. Coupling of Bone Resorption and Formation by RANKL Reverse Signalling. Nature (2018) 561:195-200. doi: 10.1038/s41586-018-0482-7

34. Cui Y, Luan J, Li H, Zhou X, Han J. Exosomes Derived From Mineralizing Osteoblasts Promote ST2 Cell Osteogenic Differentiation by Alteration of microRNA Expression. FEBS Lett (2016) 590:185-92. doi: 10.1002/18733468.12024

35. Morrell AE, Brown GN, Robinson ST, Sattler RL, Baik AD, Zhen G, et al. Mechanically Induced Ca2+ Oscillations in Osteocytes Release Extracellular Vesicles and Enhance Bone Formation. Bone Res (2018) 6:6. doi: 10.1038/ s41413-018-0007-x

36. Ren L, Song ZJ, Cai QW, Chen RX, Zou Y, Fu Q, et al. Adipose Mesenchymal Stem Cell-Derived Exosomes Ameliorate Hypoxia/Serum DeprivationInduced Osteocyte Apoptosis and Osteocyte-Mediated Osteoclastogenesis In Vitro. Biochem Biophys Res Commun (2019) 508:138-44. doi: 10.1016/ j.bbrc.2018.11.109

37. Lu Z, Chen Y, Dunstan C, Roohani-Esfahani S, Zreiqat H. Priming Adipose Stem Cells With Tumor Necrosis Factor-Alpha Preconditioning Potentiates Their Exosome Efficacy for Bone Regeneration. Tissue Eng - Part A (2017) 23:1212-20. doi: 10.1089/ten.tea.2016.0548

38. Weilner S, Keider V, Winter M, Harreither E, Salzer B, Weiss F, et al. Vesicular Galectin-3 Levels Decrease With Donor Age and Contribute to the Reduced Osteo-Inductive Potential of Human Plasma Derived Extracellular Vesicles. Aging (Albany NY) (2016) 8:16-30. doi: 10.18632/aging.100865

39. Muraca M, Cappariello A. The Role of Extracellular Vesicles (Evs) in the Epigenetic Regulation of Bone Metabolism and Osteoporosis. Int J Mol Sci (2020) 21:1-21. doi: 10.3390/ijms21228682

40. Cappariello A, Rucci N. Tumour-Derived Extracellular Vesicles (EVs): A Dangerous "Message in a Bottle" for Bone. Int J Mol Sci (2019) 20:4805-21. doi: 10.3390/ijms20194805

41. Schöneberg J, Lee IH, Iwasa JH, Hurley JH. Reverse-Topology Membrane Scission by the ESCRT Proteins. Nat Rev Mol Cell Biol (2016) 18:5-17. doi: $10.1038 / \mathrm{nrm} .2016 .121$

42. Cappariello A, Capulli M. The Vicious Cycle of Breast Cancer-Induced Bone Metastases, a Complex Biological and Therapeutic Target. Curr Mol Biol Rep (2018) 4:123-31. doi: 10.1007/s40610-018-0099-5

43. Palmerini E, Righi A, Staals EL. Rare Primary Malignant Bone Sarcomas. Cancers (Basel) (2020) 12:1-14. doi: 10.3390/cancers12113092

44. D’Oronzo S, Coleman R, Brown J, Silvestris F. Metastatic Bone Disease: Pathogenesis and Therapeutic Options. J Bone Oncol (2019) 15:004-4. doi: 10.1016/j.jbo.2018.10.004

45. Weilbaecher KN, Guise TA, McCauley LK. Cancer to Bone: A Fatal Attraction. Nat Rev Cancer (2011) 11:411-25. doi: 10.1038/nrc3055

46. Chambers AF, Groom AC, MacDonald IC. Dissemination and Growth of Cancer Cells in Metastatic Sites. Nat Rev Cancer (2002) 2:563-72. doi: $10.1038 / \mathrm{nrc} 865$

47. Croucher PI, McDonald MM, Martin TJ. Bone Metastasis: The Importance of the Neighbourhood. Nat Rev Cancer (2016) 16:373-86. doi: 10.1038/ nrc.2016.44

48. Murphey MD, Robbin MR, McRae GA, Flemming DJ, Temple HT, Kransdorf MJ. From the Archives of the AFIP: The Many Faces of Osteosarcoma. Radiographics (1997) 17:1205-31. doi: 10.1148/radiographics.17.5.9308111

49. Kundu ZS. Classification, Imaging, Biopsy and Staging of Osteosarcoma. in. Indian J Orthop (2014) 48:238-46. doi: 10.4103/0019-5413.132491

50. Lu X, Mu E, Wei Y, Riethdorf S, Yang Q, Yuan M, et al. VCAM-1 Promotes Osteolytic Expansion of Indolent Bone Micrometastasis of Breast Cancer by Engaging $\alpha 4 \beta 1$-Positive Osteoclast Progenitors. Cancer Cell (2011) 20:70114. doi: 10.1016/j.ccr.2011.11.002 
51. Brown HK, Schiavone K, Gouin F, Heymann MF, Heymann D. Biology of Bone Sarcomas and New Therapeutic Developments. Calcif Tissue Int (2018) 102:174-95. doi: 10.1007/s00223-017-0372-2

52. Jafari F, Javdansirat S, Sanaie S, Naseri A, Shamekh A, Rostamzadeh D, et al. Osteosarcoma: A Comprehensive Review of Management and Treatment Strategies. Ann Diagn Pathol (2020) 49:151654. doi: 10.1016/j.anndiagpath. 2020.151654

53. Raimondi L, De Luca A, Gallo A, Costa V, Russelli G, Cuscino N, et al. Osteosarcoma Cell-Derived Exosomes Affect Tumor Microenvironment by Specific Packaging of microRNAs. Carcinogenesis (2020) 41:666-77. doi: 10.1093/carcin/bgz130

54. Hoshino A, Costa-Silva B, Shen TL, Rodrigues G, Hashimoto A, Tesic Mark $\mathrm{M}$, et al. Tumour Exosome Integrins Determine Organotropic Metastasis. Nature (2015) 527:329-35. doi: 10.1038/nature15756

55. Wood SL, Pernemalm M, Crosbie PA, Whetton AD. The Role of the TumorMicroenvironment in Lung Cancer-Metastasis and its Relationship to Potential Therapeutic Targets. Cancer Treat Rev (2014) 40:558-66. doi: 10.1016/j.ctrv.2013.10.001

56. Taverna S, Pucci M, Giallombardo M, Di Bella MA, Santarpia M, Reclusa P, et al. Amphiregulin Contained in NSCLC-Exosomes Induces Osteoclast Differentiation Through the Activation of EGFR Pathway. Sci Rep (2017) 7:3170. doi: 10.1038/s41598-017-03460-y

57. Valencia K, Luis-Ravelo D, Bovy N, Antón I, Martínez-Canarias S, Zandueta C, et al. miRNA Cargo Within Exosome-Like Vesicle Transfer Influences Metastatic Bone Colonization. Mol Oncol (2014) 8:689-703. doi: 10.1016/ J.MOLONC.2014.01.012

58. Bragado P, Sosa MS, Keely P, Condeelis J, Aguirre-Ghiso JA. Microenvironments Dictating Tumor Cell Dormancy. Recent Results Cancer Res (2012) 195:25-39. doi: 10.1007/978-3-642-28160-0_3

59. Muzes G, Sipos F. Metastatic Cell Dormancy and Re-Activation: An Overview on Series of Molecular Events Critical for Cancer Relapse. Anticancer Agents Med Chem (2017) 17:472-82. doi: 10.2174/1871520 616666160901145857

60. Kling MJ, Chaturvedi NK, Kesherwani V, Coulter DW, McGuire TR, Sharp JG, et al. Exosomes Secreted Under Hypoxia Enhance Stemness in Ewing's Sarcoma Through miR-210 Delivery. Oncotarget (2020) 11:3633-45. doi: 10.18632/ONCOTARGET.27702

61. Zhang K, Dong C, Chen M, Yang T, Wang X, Gao Y, et al. Extracellular Vesicle-Mediated Delivery of miR-101 Inhibits Lung Metastasis in Osteosarcoma. Theranostics (2020) 10:411-25. doi: 10.7150/thno.33482

62. Prunotto M, Farina A, Lane L, Pernin A, Schifferli J, Hochstrasser DF, et al. Proteomic Analysis of Podocyte Exosome-Enriched Fraction From Normal Human Urine. JProteomics (2013) 26;82:193-229. doi: 10.1016/j.jprot.2013.01.012

63. Ono M, Kosaka N, Tominaga N, Yoshioka Y, Takeshita F, Takahashi RU, et al. Exosomes From Bone Marrow Mesenchymal Stem Cells Contain a microRNA That Promotes Dormancy in Metastatic Breast Cancer Cells. Sci Signal (2014) 7:ra63. doi: 10.1126/scisignal.2005231

64. Bliss SA, Sinha G, Sandiford OA, Williams LM, Engelberth DJ, Guiro K, et al. Mesenchymal Stem Cell-Derived Exosomes Stimulate Cycling Quiescence and Early Breast Cancer Dormancy in Bone Marrow. Cancer Res (2016) 76:5832-44. doi: 10.1158/0008-5472.CAN-16-1092

65. Walker ND, Elias M, Guiro K, Bhatia R, Greco SJ, Bryan M, et al. Exosomes From Differentially Activated Macrophages Influence Dormancy or Resurgence of Breast Cancer Cells Within Bone Marrow Stroma. Cell Death Dis (2019) 10:59-65. doi: 10.1038/s41419-019-1304-z

66. Vallabhaneni KC, Penfornis P, Xing F, Hassler Y, Adams KV, Mo YY, et al. Stromal Cell Extracellular Vesicular Cargo Mediated Regulation of Breast Cancer Cell Metastasis Via Ubiquitin Conjugating Enzyme E2 N Pathway. Oncotarget (2017) 8:109861-76. doi: 10.18632/oncotarget.22371

67. Macklin R, Wang H, Loo D, Martin S, Cumming A, Cai N, et al. Extracellular Vesicles Secreted by Highly Metastatic Clonal Variants of Osteosarcoma Preferentially Localize to the Lungs and Induce Metastatic Behaviour in Poorly Metastatic Clones. Oncotarget (2016) 7:43570-87. doi: 10.18632/ oncotarget.9781

68. Qi J, Zhou Y, Jiao Z, Wang X, Zhao Y, Li YY, et al. Exosomes Derived From Human Bone Marrow Mesenchymal Stem Cells Promote Tumor Growth Through Hedgehog Signaling Pathway. Cell Physiol Biochem (2017) 42:2242-54. doi: $10.1159 / 000479998$
69. Endo-Munoz L, Cai N, Cumming A, Macklin R, De Long LM, Topkas E, et al. Progression of Osteosarcoma From a Non-Metastatic to a Metastatic Phenotype Is Causally Associated With Activation of an Autocrine and Paracrine uPA Axis. PLoS One (2015) 10:e0133592. doi: 10.1371/journal.pone.0133592

70. Wang J, Zhang H, Sun X, Wang X, Ren T, Huang Y, et al. Exosomal PD-L1 and N-Cadherin Predict Pulmonary Metastasis Progression for Osteosarcoma Patients. J Nanobiotechnology (2020) 18:151. doi: 10.1186/ s12951-020-00710-6

71. Avnet S, Longhi A, Salerno M, Halleen JM, Perut F, Granchi D, et al. Increased Osteoclast Activity is Associated With Aggressiveness of Osteosarcoma. Int J Oncol (2008) 33:1231-8. doi: 10.3892/ijo_00000113

72. Garimella R, Washington L, Isaacson J, Vallejo J, Spence M, Tawfik O, et al. Extracellular Membrane Vesicles Derived From 143B Osteosarcoma Cells Contain Pro-Osteoclastogenic Cargo: A Novel Communication Mechanism in Osteosarcoma Bone Microenvironment. Transl Oncol (2014) 7:331-40. doi: 10.1016/j.tranon.2014.04.011

73. Ucci A, Cappariello A, Ponzetti M, Tennant F, Loftus A, Shefferd K, et al. Anti-Osteoblastogenic, Pro-Inflammatory and Pro-Angiogenic Effect of Extracellular Vesicles Isolated From the Human Osteosarcoma Cell Line Mnng/Hos. Bone (2021) 116130. doi: 10.1016/j.bone.2021.116130

74. Zöllner SK, Amatruda JF, Bauer S, Collaud S, de Álava E, DuBois SG, et al. Ewing Sarcoma-Diagnosis, Treatment, Clinical Challenges and Future Perspectives. J Clin Med (2021) 10:1685. doi: 10.3390/jcm10081685

75. Miller IV, Raposo G, Welsch U, Prazeres da Costa O, Thiel U, Lebar M, et al. First Identification of Ewing's Sarcoma-Derived Extracellular Vesicles and Exploration of Their Biological and Potential Diagnostic Implications. Biol Cell (2013) 105:289-303. doi: 10.1111/boc.201200086

76. Whelan JS, Davis LE. Osteosarcoma, Chondrosarcoma, and Chordoma. J Clin Oncol (2018) 36:188-93. doi: 10.1200/JCO.2017.75.1743

77. Cheng C, Zhang Z, Cheng F, Shao Z. Exosomal lncRNA RAMP2-AS1 Derived From Chondrosarcoma Cells Promotes Angiogenesis Through miR-2355-5p/VEGFR2 Axis. Onco Targets Ther (2020) 13:3291-301. doi: 10.2147/OTT.S244652

78. Augsburger D, Nelson PJ, Kalinski T, Udelnow A, Knösel T, Hofstetter M, et al. Current Diagnostics and Treatment of Fibrosarcoma -Perspectives for Future Therapeutic Targets and Strategies. Oncotarget (2017) 8:104638-53. doi: 10.18632/oncotarget.20136

79. Hakulinen J, Sankkila L, Sugiyama N, Lehti K, Keski-Oja J. Secretion of Active Membrane Type 1 Matrix Metalloproteinase (MMP-14) Into Extracellular Space in Microvesicular Exosomes. J Cell Biochem (2008) 105:1211-8. doi: 10.1002/jcb.21923

80. Chen Y, Di Grappa MA, Molyneux SD, McKee TD, Waterhouse P, Penninger JM, et al. RANKL Blockade Prevents and Treats Aggressive Osteosarcomas. Sci Transl Med (2015) 7:2-18. doi: 10.1126/scitranslmed.aad0295

81. Xie L, Ji T, Guo W. Anti-Angiogenesis Target Therapy for Advanced Osteosarcoma (Review). Oncol Rep (2017) 38:625-36. doi: 10.3892/ or.2017.5735

82. Jerez $\mathrm{S}$, Araya $\mathrm{H}$, Hevia D, Irarrázaval $\mathrm{CE}$, Thaler R, van Wijnen AJ, et al. Extracellular Vesicles From Osteosarcoma Cell Lines Contain miRNAs Associated With Cell Adhesion and Apoptosis. Gene (2019) 710:246-57. doi: 10.1016/J.GENE.2019.06.005

83. Wang JW, Wu XF, Gu XJ, Jiang XH. Exosomal miR-1228 From CancerAssociated Fibroblasts Promotes Cell Migration and Invasion of Osteosarcoma by Directly Targeting SCAI. Oncol Res (2019) 27:979-86. doi: $10.3727 / 096504018 X 15336368805108$

84. Qin F, Tang H, Zhang Y, Zhang Z, Huang P, Zhu J. Bone Marrow-Derived Mesenchymal Stem Cell-Derived Exosomal microRNA-208a Promotes Osteosarcoma Cell Proliferation, Migration, and Invasion. J Cell Physiol (2020) 235:4734-45. doi: 10.1002/JCP.29351

85. Wolf-Dennen K, Gordon N, Kleinerman ES. Exosomal Communication by Metastatic Osteosarcoma Cells Modulates Alveolar Macrophages to an M2 Tumor-Promoting Phenotype and Inhibits Tumoricidal Functions. Oncoimmunology (2020) 9:1747677. doi: 10.1080/2162402X.2020.1747677

86. Mazumdar A, Urdinez J, Boro A, Arlt MJE, Egli FE, Niederöst B, et al. Exploring the Role of Osteosarcoma-Derived Extracellular Vesicles in PreMetastatic Niche Formation and Metastasis in the 143-B Xenograft Mouse Osteosarcoma Model. Cancers (2020) 12:3457. doi: 10.3390/ CANCERS12113457 
87. Psaila B, Lyden D. The Metastatic Niche: Adapting the Foreign Soil. Nat Rev Cancer (2009) 9:285-93. doi: 10.1038/nrc2621

88. Sceneay J, Smyth MJ, Möller A. The Pre-Metastatic Niche: Finding Common Ground. Cancer Metastasis Rev (2013) 32:449-64. doi: 10.1007/s10555-0139420-1

89. Peinado H, Zhang H, Matei IR, Costa-Silva B, Hoshino A, Rodrigues G, et al. Pre-Metastatic Niches: Organ-Specific Homes for Metastases. Nat Rev Cancer (2017) 17:302-17. doi: 10.1038/nrc.2017.6

90. Hashimoto K, Ochi H, Sunamura S, Kosaka N, Mabuchi Y, Fukuda T, et al. Cancer-Secreted hsa-miR-940 Induces an Osteoblastic Phenotype in the Bone Metastatic Microenvironment via Targeting ARHGAP1 and FAM134A. Proc Natl Acad Sci (2018) 115:2204-9. doi: 10.1073/pnas.1717363115

91. Karlsson T, Lundholm M, Widmark A, Persson E. Tumor Cell-Derived Exosomes From the Prostate Cancer Cell Line TRAMP-C1 Impair Osteoclast Formation and Differentiation. PLoS One (2016) 11:e0166284. doi: 10.1371/journal.pone.0166284

92. Duan Y, Tan Z, Yang M, Li J, Liu C, Wang C, et al. PC-3-Derived Exosomes Inhibit Osteoclast Differentiation by Downregulating miR-214 and Blocking NF-B Signaling Pathway. BioMed Res Int (2019) 2019:1-8. doi: 10.1155/ 2019/8650846

93. Chowdhury R, Webber JP, Gurney M, Mason MD, Tabi Z, Clayton A. Cancer Exosomes Trigger Mesenchymal Stem Cell Differentiation Into ProAngiogenic and Pro-Invasive Myofibroblasts. Oncotarget (2015) 6:715-31. doi: 10.18632/oncotarget.2711

94. Abd Elmageed ZY, Yang Y, Thomas R, Ranjan M, Mondal D, Moroz K, et al. Neoplastic Reprogramming of Patient-Derived Adipose Stem Cells by Prostate Cancer Cell-Associated Exosomes. Stem Cells (2014) 32:983-97. doi: $10.1002 /$ stem.1619

95. Dai Y, Gao X. Inhibition of Cancer Cell-Derived Exosomal microRNA-183 Suppresses Cell Growth and Metastasis in Prostate Cancer by Upregulating TPM1. Cancer Cell Int (2021) 21:9-15. doi: 10.1186/s12935-020-01686-x

96. Yuan X, Qian N, Ling S, Li Y, Sun W, Li J, et al. Breast Cancer Exosomes Contribute to Pre-Metastatic Niche Formation and Promote Bone Metastasis of Tumor Cells. Theranostics (2021) 11:1429-45. doi: 10.7150/ thno.45351

97. Hannafon BN, Trigoso YD, Calloway CL, Zhao YD, Lum DH, Welm AL, et al. Plasma Exosome microRNAs are Indicative of Breast Cancer. Breast Cancer Res (2016) 18:90. doi: 10.1186/s13058-016-0753-x

98. Jung KO, Youn H, Lee CH, Kang KW, Chung JK. Visualization of ExosomeMediated miR-210 Transfer From Hypoxic Tumor Cells. Oncotarget (2017) 8:9899-910. doi: 10.18632/oncotarget.14247

99. Luga V, Zhang L, Viloria-Petit AM, Ogunjimi AA, Inanlou MR, Chiu E, et al. Exosomes Mediate Stromal Mobilization of Autocrine Wnt-PCP Signaling in Breast Cancer Cell Migration. Cell (2012) 151:1542-56. doi: 10.1016/ j.cell.2012.11.024

100. Loftus A, Cappariello A, George C, Ucci A, Shefferd K, Green A, et al. Extracellular Vesicles From Osteotropic Breast Cancer Cells Affect Bone Resident Cells. J Bone Miner Res (2019) 35:396-412. doi: 10.1002/jbmr.3891

101. Damsky WE, Theodosakis N, Bosenberg M. Melanoma Metastasis: New Concepts and Evolving Paradigms. Oncogene (2014) 33:2413-22. doi: 10.1038/onc.2013.194

102. Zekri J, Marples M, Taylor D, Kandukurti K, McParland L, Brown JE. Complications of Bone Metastases From Malignant Melanoma. J Bone Oncol (2017) 8:13-7. doi: 10.1016/j.jbo.2017.08.003

103. Mannavola F, Tucci M, Felici C, Passarelli A, D’Oronzo S, Silvestris F. Tumor-Derived Exosomes Promote the In Vitro Osteotropism of Melanoma Cells by Activating the SDF-1/CXCR4/CXCR7 Axis. J Transl Med (2019) 17:230. doi: 10.1186/s12967-019-1982-4

104. Sosa MS, Bragado P, Aguirre-Ghiso JA. Mechanisms of Disseminated Cancer Cell Dormancy: An Awakening Field. Nat Rev Cancer (2014) 14:611-22. doi: $10.1038 /$ nrc3793

105. Linde N, Fluegen G, Aguirre-Ghiso JA. "The Relationship Between Dormant Cancer Cells and Their Microenvironment," in. Adv Cancer Res (2016) 132:45-71. doi: 10.1016/bs.acr.2016.07.002

106. Capulli M, Hristova D, Valbret Z, Carys K, Arjan R, Maurizi A, et al. Notch2 Pathway Mediates Breast Cancer Cellular Dormancy and Mobilisation in Bone and Contributes to Haematopoietic Stem Cell Mimicry. Br J Cancer (2019) 121:157-71. doi: 10.1038/s41416-019-0501-y
107. Chaffer CL, Weinberg RA. A Perspective on Cancer Cell Metastasis. Science (2011) 331:1559-64. doi: 10.1126/science.1203543

108. Lv MM, Zhu XY, Chen WX, Zhong SL, Hu Q, Ma TF, et al. Exosomes Mediate Drug Resistance Transfer in MCF-7 Breast Cancer Cells and a Probable Mechanism is Delivery of P-Glycoprotein. Tumor Biol (2014) 35:10773-9. doi: 10.1007/s13277-014-2377-z

109. Wang W, Zou L, Zhou D, Zhou Z, Tang F, Xu Z, et al. Overexpression of Ubiquitin Carboxyl Terminal Hydrolase-L1 Enhances Multidrug Resistance and Invasion/Metastasis in Breast Cancer by Activating the MAPK/Erk Signaling Pathway. Mol Carcinog (2016) 55:1329-42. doi: 10.1002/mc.22376

110. Wei Y, Lai X, Yu S, Chen S, Ma Y, Zhang Y, et al. Exosomal miR-221/222 Enhances Tamoxifen Resistance in Recipient ER-Positive Breast Cancer Cells. Breast Cancer Res Treat (2014) 147:423-31. doi: 10.1007/s10549014-3037-0

111. Yang SJ, Wang DD, Li J, Xu HZ, Shen HY, Chen X, et al. Predictive Role of GSTP1-Containing Exosomes in Chemotherapy-Resistant Breast Cancer. Gene (2017) 623:5-14. doi: 10.1016/J.GENE.2017.04.031

112. Kreger B, Johansen E, Cerione R, Antonyak M. The Enrichment of Survivin in Exosomes From Breast Cancer Cells Treated With Paclitaxel Promotes Cell Survival and Chemoresistance. Cancers (Basel) (2016) 8:111. doi: 10.3390/cancers8120111

113. Ciravolo V, Huber V, Ghedini GC, Venturelli E, Bianchi F, Campiglio M, et al. Potential Role of HER2-Overexpressing Exosomes in Countering Trastuzumab-Based Therapy. J Cell Physiol (2012) 227:658-67. doi: $10.1002 /$ jcp. 22773

114. Boelens MC, Wu TJ, Nabet BY, Xu B, Qiu Y, Yoon T, et al. Exosome Transfer From Stromal to Breast Cancer Cells Regulates Therapy Resistance Pathways. Cell (2014) 159:499-513. doi: 10.1016/j.cell.2014.09.051

115. Shan G, Gu J, Zhou D, Li L, Cheng W, Wang Y, et al. Cancer-Associated Fibroblast-Secreted Exosomal miR-423-5p Promotes Chemotherapy Resistance in Prostate Cancer by Targeting GREM2 Through the TGF- $\beta$ Signaling Pathway. Exp Mol Med (2020) 52:1809-22. doi: 10.1038/s12276020-0431-z

116. Moon PG, Lee JE, Cho YE, Lee SJ, Chae YS, Jung JH, et al. Fibronectin on Circulating Extracellular Vesicles as a Liquid Biopsy to Detect Breast Cancer. Oncotarget (2016) 7:40189-99. doi: 10.18632/oncotarget.9561

117. Shimizu T, Sugihara E, Yamaguchi-Iwai S, Tamaki S, Koyama Y, Kamel W, et al. IGF2 Preserves Osteosarcoma Cell Survival by Creating an Autophagic State of Dormancy That Protects Cells Against Chemotherapeutic Stress. Cancer Res (2014) 74:6531-41. doi: 10.1158/0008-5472.CAN-14-0914

118. Casson J, Davies OG, Smith CA, Dalby MJ, Berry CC. Mesenchymal Stem Cell-Derived Extracellular Vesicles may Promote Breast Cancer Cell Dormancy. J Tissue Eng (2018) 9:1-7. doi: 10.1177/2041731418810093

119. Moon PG, Lee JE, Cho YE, Lee SJ, Jung JH, Chae YS, et al. Identification of Developmental Endothelial Locus-1 on Circulating Extracellular Vesicles as a Novel Biomarker for Early Breast Cancer Detection. Clin Cancer Res (2016) 22:1757-66. doi: 10.1158/1078-0432.CCR-15-0654

120. Vardaki I, Ceder S, Rutishauser D, Baltatzis G, Foukakis T, Panaretakis T. Periostin is Identified as a Putative Metastatic Marker in Breast Cancer-Derived Exosomes. Oncotarget (2016) 7:74966-78. doi: 10.18632/oncotarget.11663

121. Fang S, Tian H, Li X, Jin D, Li X, Kong J, et al. Clinical Application of a Microfluidic Chip for Immunocapture and Quantification of Circulating Exosomes to Assist Breast Cancer Diagnosis and Molecular Classification. PLoS One (2017) 12:e0175050. doi: 10.1371/journal.pone.0175050

122. Sandfeld-Paulsen B, Jakobsen KR, Bæk R, Folkersen BH, Rasmussen TR, Meldgaard P, et al. Exosomal Proteins as Diagnostic Biomarkers in Lung Cancer. J Thorac Oncol (2016) 11:1701-10. doi: 10.1016/j.jtho.2016.05.034

123. Eichelser C, Stückrath I, Müller V, Milde-Langosch K, Wikman H, Pantel K, et al. Increased Serum Levels of Circulating Exosomal microRNA-373 in Receptor-Negative Breast Cancer Patients. Oncotarget (2014) 5:9650-63. doi: 10.18632/oncotarget.2520

124. Bryant RJ, Pawlowski T, Catto JWF, Marsden G, Vessella RL, Rhees B, et al. Changes in Circulating microRNA Levels Associated With Prostate Cancer. Br J Cancer (2012) 106:768-74. doi: 10.1038/bjc.2011.595

125. Alegre E, Sanmamed MF, Rodriguez C, Carranza O, Martín-Algarra S, González Á. Study of Circulating MicroRNA-125b Levels in Serum Exosomes in Advanced Melanoma. Arch Pathol Lab Med (2014) 138:82832. doi: 10.5858/arpa.2013-0134-OA 
126. Luan X, Sansanaphongpricha K, Myers I, Chen H, Yuan H, Sun D. Engineering Exosomes as Refined Biological Nanoplatforms for Drug Delivery. Acta Pharmacol Sin (2017) 38:754-63. doi: 10.1038/aps.2017.12

127. Melzer C, Rehn V, Yang Y, Bähre H, von der Ohe J, Hass R. Taxol-Loaded MSC-Derived Exosomes Provide a Therapeutic Vehicle to Target Metastatic Breast Cancer and Other Carcinoma Cells. Cancers (Basel) (2019) 11:798. doi: 10.3390/cancers 11060798

128. Haney MJ, Zhao Y, Jin YS, Li SM, Bago JR, Klyachko NL, et al. MacrophageDerived Extracellular Vesicles as Drug Delivery Systems for Triple Negative Breast Cancer (TNBC) Therapy. J Neuroimmune Pharmacol (2020) 15:487500. doi: 10.1007/s11481-019-09884-9

129. Naseri M, Bozorgmehr M, Zöller M, Ranaei Pirmardan E, Madjd Z. TumorDerived Exosomes: The Next Generation of Promising Cell-Free Vaccines in Cancer Immunotherapy. Oncoimmunology (2020) 9:91-110. doi: 10.1080/ 2162402X.2020.1779991

130. Zhang Y, Luo CL, He BC, Zhang JM, Cheng GZ, Wu XH. Exosomes Derived From IL-12-Anchored Renal Cancer Cells Increase Induction of Specific Antitumor Response In Vitro: A Novel Vaccine for Renal Cell Carcinoma. Int J Oncol (2010) 36:133-40. doi: 10.3892/ijo-00000484

131. Zheng HC. The Molecular Mechanisms of Chemoresistance in Cancers. Oncotarget (2017) 8:59950-64. doi: 10.18632/oncotarget.19048

132. Torreggiani E, Roncuzzi L, Perut F, Zini N, Baldini N. Multimodal Transfer of MDR by Exosomes in Human Osteosarcoma. Int J Oncol (2016) 49:18996. doi: 10.3892/ijo.2016.3509

133. Yoshida A, Fujiwara T, Uotani K, Morita T, Kiyono M, Yokoo S, et al. Clinical and Functional Significance of Intracellular and Extracellular microRNA-25-3p in Osteosarcoma. Acta Med Okayama (2018) 72:165-74. doi: 10.18926/AMO/55857

134. Weinman MA, Ramsey SA, Leeper HJ, Brady JV, Schlueter A, Stanisheuski S, et al. Exosomal Proteomic Signatures Correlate With Drug Resistance and Carboplatin Treatment Outcome in a Spontaneous Model of Canine Osteosarcoma. Cancer Cell Int (2021) 21:245. doi: 10.1186/s12935-02101943-7

135. Pan Y, Lin Y, Mi C. Cisplatin-Resistant Osteosarcoma Cell-Derived Exosomes Confer Cisplatin Resistance to Recipient Cells in an Exosomal Circ_103801-Dependent Manner. Cell Biol Int (2021) 45(4):858-68. doi: $10.1002 /$ cbin.11532

136. Chen W-X, Wang D-D, Zhu B, Zhu Y-Z, Zheng L, Feng Z-Q, et al. Exosomal miR-222 From Adriamycin-Resistant MCF-7 Breast Cancer Cells Promote Macrophages M2 Polarization via PTEN/Akt to Induce Tumor Progression. Aging (Albany NY) (2021) 13:10415-30. doi: 10.18632/aging.202802

137. Logozzi M, Angelini DF, Giuliani A, Mizzoni D, Di Raimo R, Maggi M, et al. Increased Plasmatic Levels of PSA-Expressing Exosomes Distinguish Prostate Cancer Patients From Benign Prostatic Hyperplasia: A Prospective Study. Cancers (Basel) (2019) 11:1449. doi: 10.3390/cancers11101449

138. Gabriel K, Ingram A, Austin R, Kapoor A, Tang D, Majeed F, et al. Regulation of the Tumor Suppressor PTEN Through Exosomes:
A Diagnostic Potential for Prostate Cancer. PloS One (2013) 8:e70047. doi: 10.1371/journal.pone.0070047

139. Logozzi M, De Milito A, Lugini L, Borghi M, Calabrò L, Spada M, et al. High Levels of Exosomes Expressing CD63 and Caveolin-1 in Plasma of Melanoma Patients. PLoS One (2009) 4:e5219. doi: 10.1371/journal.pone.0005219

140. Xu Y, Liu Y, Yang C, Kang L, Wang M, Hu J, et al. Macrophages Transfer Antigens to Dendritic Cells by Releasing Exosomes Containing Dead-CellAssociated Antigens Partially Through a Ceramide-Dependent Pathway to Enhance CD4+ T-Cell Responses. Immunology (2016) 149:157-71. doi: $10.1111 / \mathrm{imm} .12630$

141. Tian X, Zhu M, Tian Y, Ramm GA, Zhao Y, Nie G. A Membrane VesicleBased Dual Vaccine Against Melanoma and Lewis Lung Carcinoma. Biomaterials (2012) 33:6147-54. doi: 10.1016/j.biomaterials.2012.05.034

142. Zhu L, Kalimuthu S, Oh JM, Gangadaran P, Baek SH, Jeong SY, et al. Enhancement of Antitumor Potency of Extracellular Vesicles Derived From Natural Killer Cells by IL-15 Priming. Biomaterials (2019) 190-191:38-50. doi: 10.1016/j.biomaterials.2018.10.034

143. Zampetaki A, Mayr M. Analytical Challenges and Technical Limitations in Assessing Circulating MiRNAs. Thromb Haemost (2012) 108:592-8. doi: 10.1160/TH12-02-0097

144. Ludwig N, Whiteside TL, Reichert TE. Challenges in Exosome Isolation and Analysis in Health and Disease. Int J Mol Sci (2019) 20:4684-94. doi: 10.3390/ijms20194684

145. Witwer KW. XenomiRs and miRNA Homeostasis in Health and Disease: Evidence That Diet and Dietary miRNAs Directly and Indirectly Influence Circulating miRNA Profiles. RNA Biol (2012) 9:1147-54. doi: 10.4161/ rna.21619

146. Shende VR, Goldrick MM, Ramani S, Earnest DJ. Expression and Rhythmic Modulation of Circulating MicroRNAs Targeting the Clock Gene Bmall in Mice. PLoS One (2011) 6:e22586. doi: 10.1371/journal.pone.0022586

Conflict of Interest: The authors declare that the research was conducted in the absence of any commercial or financial relationships that could be construed as a potential conflict of interest.

Publisher's Note: All claims expressed in this article are solely those of the authors and do not necessarily represent those of their affiliated organizations, or those of the publisher, the editors and the reviewers. Any product that may be evaluated in this article, or claim that may be made by its manufacturer, is not guaranteed or endorsed by the publisher.

Copyright $\odot 2021$ Cappariello and Rucci. This is an open-access article distributed under the terms of the Creative Commons Attribution License (CC BY). The use, distribution or reproduction in other forums is permitted, provided the original author(s) and the copyright owner(s) are credited and that the original publication in this journal is cited, in accordance with accepted academic practice. No use, distribution or reproduction is permitted which does not comply with these terms. 